\title{
¿EL SEXISMO Y LA AUTOESTIMA SEXUAL GUARDAN RELACIÓN? ANÁLISIS DE LAS DIFERENCIAS DE GÉNERO
}

\author{
Verónica Estruch-García \\ Dpto de Psicología Evolutiva y de la Educación \\ Universitat de València \\ Vesgar2@alumni.uv.es \\ Olga Fernández-García \\ Dpto de Psicología Evolutiva y de la Educación \\ Universitat de València \\ María Dolores Gil-Llario \\ Dpto de Psicología Evolutiva y de la Educación \\ Universitat de València
}

Recepción Artículo: 13 mayo 2021

Admisión Evaluación: 13 mayo 2021

Informe Evaluador 1: 26 mayo 2021

Informe Evaluador 2: 28 mayo 2021

Aprobación Publicación: 01 junio 2021

\section{RESUMEN}

Las creencias sexistas se originan ante la percepción de diferencias entre hombres y mujeres respecto a características biológicas y sociales. Se trata de un constructo bidimensional compuesto por el sexismo hostil, que implica actitudes abiertamente negativas hacia las mujeres, y el sexismo benévolo, que se caracteriza por un tono afectivo positivo que idealiza a las mujeres al tiempo que enfatiza su debilidad. Ambos tipos de sexismo son perjudiciales y contribuyen a mantener las desigualdades sociales, por lo que es necesario conocer aquellas variables que pueden estar relacionadas con este tipo de actitudes. En este sentido, la autoestima sexual parece actuar como una variable predictora de actitudes más negativas hacia las mujeres. Por ello, el objetivo de este estudio fue explorar la relación existente entre las dos dimensiones del sexismo, y la autoestima sexual en hombres y mujeres. Un total de 252 personas, de 18 a 69 años (68,25\% fueron mujeres; $31,75 \%$ fueron hombres) participaron en el estudio. Los resultados sugieren que existen diferencias significativas entre hombres y mujeres en sexismo hostil $(t=-3,933 ; p=0,000)$ y en sexismo benévolo ( $t=-$ 4,$470 ; p=0,000$ ), siendo los hombres quienes presentan en mayor medida ambos tipos de sexismo. Por otra parte, parece que existe una relación positiva y estadísticamente significativa entre sexismo benévolo y la autoestima sexual, únicamente en los hombres ( $r=0,362 ; p=0,001)$, mostrando el análisis de regresión que la autoestima sexual alcanza a explicar un $13,1 \%$ del sexismo benévolo. 


\section{¿EL SEXISMO Y LA AUTOESTIMA SEXUAL GUARDAN RELACIÓN? ANÁLISIS DE LAS DIFERENCIAS DE GÉNERO}

En conclusión, nuestro estudio constata que la autoestima sexual y el sexismo benevolente son dos variables que, en el caso de los hombres, se hallan fuertemente relacionadas.

Palabras clave: actitudes sexistas; sexismo hostil; sexismo benevolente; autoestima sexual

\section{ABSTRACT}

Are sexism and sexual self-esteem related? analysis of gender differences. The perception of differences between men and women regarding biological and social characteristics are the origin sexist beliefs. This is a two-dimensional construct that it is composed of hostile sexism implies openly negative attitudes towards women, and benevolent sexism is characterized by a positive affective tone that idealizes women while emphasizing their weakness. Both types of sexism are harmful and contribute to maintain social inequalities, so it is necessary to know those variables that may be related to this type of attitudes. In this sense, sexual self-esteem seems to act as a predictor variable of more negative attitudes towards women. Therefore, the objective of this study was to explore the relationship between the two dimensions of sexism and sexual self-esteem in men and women. A total of 252 people participated in the study (of which $68.25 \%$ were women; $31.75 \%$ were men) are 18 to 69 years. The results suggest that there are significant differences between men and women in hostile sexism ( $t=-3.933 ; p=0.000)$ and in benevolent sexism $(t=-4.470 ; p=0.000)$, the men presented both types of sexism. On the other hand, it seems that there is a positive and statistically significant relationship between benevolent sexism and sexual self-esteem, only in men $(r$ $=0.362 ; p=0.001$ ), the regression analysis showing that sexual self-esteem explains $13,1 \%$ of benevolent sexism. In conclusion, our study confirms that sexual self-esteem and benevolent sexism are two variables that, in the case of men, are strongly related.

Keywords: sexist attitudes; hostile sexism; benevolent sexism; sexual self-esteem

\section{INTRODUCCIÓN}

Las personas somos incapaces de procesar toda la información del exterior, por ello, se emplean los heurísticos, que son atajos mentales que permiten responder a una situación con un procesamiento cognitivo mínimo (Martínez, 2018). En este sentido, los estereotipos de género funcionan como heurísticos (Macrae et al., 1994) y, de acuerdo con ellos, se atribuyen características físicas, psicológicas y sociales a un género en concreto (Eagly, 1987). Los atributos asignados al género masculino han sido socialmente mejor valorados, debido a que, durante muchas décadas los hombres han representado los roles más altos en la jerarquía social, mientras que a las mujeres se les ha asignado estereotipos que se perciben como más insignificantes. El sexismo está influenciado por los estereotipos de género, pues se acepta o rechaza a una persona en función de la congruencia entre su comportamiento y las características atribuidas al género al que pertenece y a los roles sociales del mismo (De Lemus et al., 2008; Lee et al., 2010).

Glick y Fiske (1996) definieron el sexismo como un constructo bidimensional, originado en la percepción de aspectos biológicos y sociales, como el patriarcado, la diferenciación de géneros y la sexualidad (González-Rivera \& Díaz-Loving, 2018). Por una parte, plantearon la existencia del sexismo hostil, que implica actitudes negativas hacia las mujeres como consecuencia de la necesidad de dominación masculina, y tiene su origen en la percepción de las diferencias de género como una amenaza que justifica la relación de competitividad entre hombres y mujeres. Así pues, las personas con altos niveles de sexismo hostil atribuyen a los rasgos de las mujeres un matiz de peligrosidad, debido al poder diádico que estos atributos les conceden. Por otra parte, el sexismo benévolo implica actitudes más positivas hacia las mujeres, basándose en la atribución de rasgos y roles deseables al género femenino, ya que se perciben como complementarios a los roles masculinos, lo que genera una relación de interdependencia. Ambos son fuente de discriminación (Ramiro- 
Sánchez et al., 2018) y están presentes en diferentes culturas (Glick et al., 2000; Glick \& Fiske, 2001; Lee et al., 2010), aunque el sexismo benévolo puede ser un prejuicio más peligroso, debido a que las evaluaciones positivas son una herramienta para mantener la sumisión de las mujeres (De Lemus et al., 2008; Lee et al., 2010; Soto-Quevedo, 2012).

Estudios recientes sobre este tema se han centrado en conocer las variables que podrían predecir las actitudes sexistas (León \& Aizpurúa, 2020). En este sentido, la mayoría de investigaciones hayan que los hombres obtienen puntuaciones más altas que las mujeres tanto en sexismo hostil como en benévolo(Arbach et al., 2019; De Lemus et al., 2008; Fernández-García et al., 2020; Glick et al., 2000; León \& Aizpurúa, 2020; Zubieta et al., 2011); sin embargo, en otras investigaciones solo se han hallado diferencias entre hombres y mujeres en sexismo hostil, pero no en benévolo, siendo éste último más aceptado en hombres y en mujeres (Glick \& Fiske, 2001; Novo et al., 2016). Por otra parte, puntuaciones altas en ambos tipos de sexismos han sido asociadas a personas que han ejercido 0 sufrido violencia por parte de la pareja (Arbach et al., 2019), lo que destaca la importancia de conocer qué factores podrían ser buenos predictores de este tipo de actitudes. En esta línea, Sibley \& Wilson, (2004) sugirieron que el autoconcepto sexual podría ser un factor moderador del sexismo hostil y el sexismo benévolo, al influir en la forma de percibir y procesar la información de otras personas 0 de las relaciones personales (Pai et al., 2012). Así, los hombres con un esquemasexual positivo tendían a procesar la información mediante los estereotipos de género, aumentando Ios niveles de hostilidad hacia ellas. La autoestima sexual es la confianza que tiene una personas sobre la propia capacidad para disfrutar de la sexualidad de forma saludable, positiva y satisfactoria (Torres-Obregon et al., 2019), como resultado de evaluar los propios atributos físicos y no físicos (Amos \& McCabe, 2015). No obstante, Snell y Papini (1989) refieren que la autoestima sexual es la evaluación que realiza un individuo de la propia capacidad para complacer sexualmente a una pareja, destacando así la implicación de la pareja en la obtención de una buena autoestima sexual. Snell, Fisher y Walters (1993) examinaron esta dimensión en una muestra de estudiantes universitarios y hallaron que los hombres presentan mayor autoestima sexual que las mujeres. Por otra parte, Fowers \& Fowers (2010), hallaron que los hombres con menor autoestima sexual parecían mostrar altos niveles de sexismo benévolo hacia las mujeres que se comportaban de forma ajustada al rol tradicional (casta), mientras que no mostraban este tipo de actitudes hacia las mujeres que contradicen tal rol. Por otra parte, hallaron que el autoesquema sexual en las mujeres interactuaba con el sexismo hostil, y evaluaban de forma más negativa a aquellas mujeres que representan un comportamiento más promiscuo.

Tras revisar la literatura existente, se constata que son escasos los estudios que analizan la relación entre autoestima sexual y creencias sexistas. Así pues, teniendo en cuenta las desventajosas consecuencias que se derivan de las actitudes sexistas, también a nivel sexual, y con la finalidad de aportar evidencia empírica sobre factores que podrían estar asociados al sexismo, la presente investigación se plantea tres objetivos. Por un lado, explorar las diferencias entre hombres y mujeres en autoestima sexual y en ambos tipos de sexismo (hostil y benévolo), esperando hallar diferencias en las actitudes hostiles y benévolas y en autoestima sexual. Por otra parte, analizar las relaciones entre autoestima sexual y ambos tipos de sexismo, esperando encontrar una relación positiva entre sexismo hostil y autoestima sexual, y una relación negativa entre sexismo benévolo y autoestima sexual. Y, por último, explorar la influencia de la autoestima sexual sobre el sexismo hostil y benévolo, esperando que la autoestima sexual funcione como un buen predictor de ambos tipos de sexismo.

\section{MÉTODO \\ Participantes}

La muestra está formada por 252 personas de la provincia de Valencia (España), de las cuales 


\section{¿EL SEXISMO Y LA AUTOESTIMA SEXUAL GUARDAN RELACIÓN? ANÁLISIS DE LAS DIFERENCIAS DE GÉNERO}

172 son mujeres $(68,25 \%)$ y 80 son hombres $(31,25 \%)$, con edades comprendidas entre los 18 y los 69 años ( $x=33,42$, DT=12,60).

En cuanto a las mujeres, la media de edad fue de 33,02 (DT=12,622). Respecto a la orientación sexual, el $84,65 \%$ de las mujeres se identifican como heterosexuales, el 1,8\% como homosexuales y el $13,6 \%$ como bisexuales. En lo referente al estado civil, el $34,9 \%$ estaban solteras, el $54,1 \%$ casadas o en pareja, el 9,3\% separadas o divorciadas y el 1,7\% viudas. En cuanto al nivel de estudios, el 0,7\% de las mujeres no tenía estudios, el 9,3\% tenía estudios primarios, el 22,9\% estudios secundarios, el 28,9\% había realizado estudios de formación profesional, el 25,9\% eran graduados y el $12 \%$ tenían estudios de postgrado.

En referencia a la muestra de hombres, la media de edad fue de 34,29 años (DT=12,592). Respecto a la orientación sexual, el $87,3 \%$ de los hombres se identifican como heterosexuales, el $5,1 \%$ como homosexuales y el 7,6\% como bisexuales. En lo referente al estado civil, el $51,1 \%$ estaban solteros, el $43,8 \%$ casados 0 en pareja, el 3,8\% separados o divorciados y 1,3\% viudos. En cuanto al nivel de estudios, el 2,7\% de los hombres no tenía estudios, el 9,5\% tenía estudios primarios, el $27 \%$ estudios secundarios, el 29,7\% había cursado formación profesional, el 16,2\% eran graduados y el $14,9 \%$ tenían estudios de postgrado.

\section{Instrumentos}

Subescala de Autoestima Sexual del Cuestionario Multidimensional sobre Sexualidad (Snell et al., 1993). Esta subescala evalúa la percepción que tiene la persona de su valía en el ámbito sexual ("Soy una pareja sexual bastante buena"). Está formada por 5 ítems con un formato de respuestas tipo Likert de 5 niveles, que van desde el 1 (no me caracteriza) al 5 (me caracteriza mucho). A efectos interpretativos, una mayor puntuación sugiere una mayor autoestima sexual, siendo la puntuación mínima 5 y la máxima 25. La consistencia interna obtenida en autoestima sexual es buena, con un Alfa de Cronbach de 0,837.

Inventario de Sexismo Ambivalente (Glick y Fiske, 1996; Lemus, et al. 2008). Este cuestionario está formado por 20 ítems, agrupados en dos subescalas: sexismo hostil, formado por los 10 primeros ítems del inventario ( $p$. ej. "Los chicos deben controlar con quién se relacionan sus novias") y, la subescala de sexismo benévolo, compuesto por los últimos 10 ítems (p. ej. "Por las noches los chicos deben acompañar a las chicas hasta su casa para que no les ocurra nada malo"). Los ítems presentan un formato de respuesta tipo Likert con 6 alternativas, desde 1 (muy en desacuerdo) a 6 (muy de acuerdo). Todos los reactivos tienen una formulación positiva, siendo la puntuación mínima que se puede obtener en cada escala 10 y la máxima 60 , entendiendo que, a mayor puntuación, mayor presencia de actitudes sexistas. La consistencia interna de las dos subescalas es buena, siendo el Alfa de Cronbach en sexismo hostil de 0,828 y, en la subescala de sexismo benévolo de 0,793 .

\section{Procedimiento}

La investigación se llevó a cabo en el periodo de tiempo comprendido entre los meses de enero y abril del 2020. En primer lugar, se realizó una exhaustiva revisión bibliográfica de las variables que se estudian en la presente investigación con el objetivo de obtener una definición clara de los diferentes constructos, y se presentó el proyecto al Comité de Ética de Investigación de la Universitat de València. Una vez el proyecto fue aprobado, se elaboró y distribuyó la batería de ítems en formato online mediante la plataforma LimeSurvey. Para la difusión de la investigación se emplearon, sobre todo, las redes sociales (Facebook, Instagram), y también otros canales de comunicación como Gmail, mediante la técnica de la bola de nieve, pidiéndole a los amigos y conocidos que la enviaran 
a tres de sus contactos. La metodología empleada para la recogida de datos cumplía todos los requisitos éticos, ya que, previa a la cumplimentación de los ítems, se informaba a los participantes de las condiciones del estudio (objetivo, tiempo de duración, voluntariedad y anonimato, así como libertad para abandonar la cumplimentación de los instrumentos en cualquier momento si se deseaba), y debían aceptar una cláusula de consentimiento informado. Además, se introdujo un parámetro de control de edad mínima de 18 años para cumplimentarlo. Para evitar la falsedad de datos se eliminaron aquellos sujetos que no habían cumplimentado la batería completa. Al finalizar la recogida de datos, se realizaron las transformaciones de los datos pertinentes para extraer los factores de cada variable estudiada por los instrumentos de evaluación y se procedió al análisis de resultados mediante el programa de estadístico SPSS versión 19.

\section{Análisis estadísticos}

Atendiendo a los objetivos de la presente investigación, en primer lugar, con la finalidad de explorar las diferencias existentes entre hombres y mujeres en autoestima sexual y sexismo, se realizó la prueba t Student para muestras independientes. Por otra parte, con el objetivo de explorar las relaciones entre las variables estudiadas se realizó un análisis de correlaciones bivariadas de Pearson. Y, por último, se realizó un análisis de regresión múltiple para analizar la influencia de la autoestima sexual sobre el sexismo hostil y benévolo. En todos los casos la significación estadística se estableció en 0,05 .

\section{RESULTADOS}

En primer lugar, se exponen los resultados obtenidos al realizar la comparación de medias en las variables estudiadas en cuanto al género. Como se puede observar en la Tabla 1, en lo que se refiere al sexismo hostil, encontramos diferencias estadísticamente significativas ( $t=-3,93 ; p=$ 0,$000 ; d=0,47$ ), obteniendo los hombres una puntuación media de 26,61 (DT=9,60) y, las mujeres una puntuación media de 21,70 (DT=9,02). En cuanto al factor sexismo benévolo $(t=-4,47 ; p=$ $0,000 ; d=-0,53)$, los hombres obtuvieron puntuaciones más altas $(X=30,96 ; D T=10,35)$ en comparación con las mujeres $(X=25,06$; $D T=9,46)$. En autoestima sexual no se hayan diferencias estadísticamente significativas entre hombres y mujeres $(X=17,81$, DT $=4,82 ; X=17,58$, DT $=4,50$, respectivamente).

Tabla 1. Comparación de las variables del estudio por sexo.

\begin{tabular}{cccccc}
\hline & $\begin{array}{c}\text { Hombres } \\
\bar{X}(\mathrm{DT})\end{array}$ & $\begin{array}{c}\text { Mujeres } \\
\bar{X}(\mathrm{DT})\end{array}$ & $\mathrm{T}$ & $\mathrm{p}$ & $\mathrm{d}$ \\
& & & & & \\
\hline Autoestima sexual & $17,812(4,826)$ & $17,587(4,508)$ &,- 361 &, 718 &,- 012 \\
Sexismo hostil & $26,612(9,609)$ & $21,709(9,022)$ & $-3,933$ &, 000 &,- 487 \\
Sexismo benévolo & $30,962(10,350)$ & $25,064(9,460)$ & $-4,470$ &, 000 &,- 555 \\
\hline
\end{tabular}

Para dar respuesta al segundo objetivo de la investigación, se van a exponer los resultados obtenidos en el análisis de correlaciones de los dos tipos de sexismo y la autoestima sexual. Debido a las diferencias de género halladas en las variables anteriores, se ha considerado adecuado realizar el análisis de correlaciones segmentando la muestra en hombres y mujeres. Tal y como se puede observar en la Tabla 2, la autoestima sexual correlacionó de forma positiva y estadísticamente sig- 


\section{¿EL SEXISMO Y LA AUTOESTIMA SEXUAL GUARDAN RELACIÓN? ANÁLISIS DE LAS DIFERENCIAS DE GÉNERO}

nificativa con sexismo benévolo en hombres $(r=0,362 ; p=0,001)$, aunque no en mujeres $(r=0,110$; $\mathrm{p}=0,152)$. Por otra parte, la autoestima sexual no apreció correlacionada de forma significativa con sexismo hostil ni en hombres $(r=0,174 ; p=0,123)$ ni en mujeres $(r=-0,011 ; p=0,884)$.

Tabla 2. Correlaciones bivariadas

\begin{tabular}{lcccc}
\cline { 2 - 5 } & \multicolumn{2}{c}{ Hombres } & \multicolumn{2}{c}{ Mujeres } \\
\cline { 2 - 5 } & Sexismo hostil & $\begin{array}{c}\text { Sexismo } \\
\text { benévolo }\end{array}$ & $\begin{array}{c}\text { Sexismo } \\
\text { hostil }\end{array}$ & $\begin{array}{c}\text { Sexismo } \\
\text { benévolo }\end{array}$ \\
\hline Autoestima sexual &, 174 &, $362^{* *}$ &,- 011 &, 110 \\
\hline$* * \mathrm{p}=0,001$ & & & &
\end{tabular}

En lo referido al tercer objetivo, obtener evidencias sobre la validez de las inferencias que podemos realizar a partir de las dimensiones estudiadas, se realizó el análisis únicamente teniendo en cuenta aquellas variables que aparecieron correlacionadas de forma estadísticamente significativa. Como puede observarse en la Tabla 3, el modelo de regresión lineal formado por autoestima sexual explicó el $13,1 \%\left(R^{2}\right.$ ajustado $\left.=0,131\right)$ de la varianza del sexismo benévolo en hombres, siendo esta variable estadísticamente significativa $(t=3,429 ; p=0,001)$. En este modelo, por cada unidad de incremento en autoestima sexual, el sexismo benévolo aumenta 0,362 unidades.

El nivel de tolerancia y los factores de inflación de la varianza indicaron bajo riesgo de multicolinealidad en ambos modelos. El valor Durbin-Watson verificó la independencia de los errores $(\mathrm{DW}=1,724)$.

Tabla 3. Modelo de regresión lineal de sexismo benévolo en hombres.

\begin{tabular}{ccccccccc}
\hline $\begin{array}{c}\text { Variables } \\
\text { incluidas en } \\
\text { el modelo }\end{array}$ & $\begin{array}{c}\text { Modelo } \\
\text { de } \\
\text { regresión }\end{array}$ & $\begin{array}{c}\text { Coeficientes } \\
\text { no } \\
\text { estandarizado } \\
\mathrm{s}\end{array}$ & $\begin{array}{c}\text { Coeficientes } \\
\text { estandarizados }\end{array}$ & & $\begin{array}{c}\text { Estadísticas de } \\
\text { colinealidad }\end{array}$ \\
\cline { 2 - 9 } & $\mathrm{R}_{\text {ajustado }}^{2}$ & $\mathrm{~B}$ & Beta & $\mathrm{t}$ & $\mathrm{p}$ & Tolerancia & $\mathrm{VIF}$ \\
\hline $\begin{array}{c}\text { Autoestima } \\
\text { sexual }\end{array}$ &, 131 &, 776 &, 362 & 3,429 &, 001 & 1 & 1 \\
\hline
\end{tabular}

\section{DISCUSIÓN}

La finalidad de la presente investigación fue ampliar el conocimiento sobre los factores predictores del sexismo hostil y benévolo. Respecto al primer objetivo, los resultados parecen sugerir que, se confirma la primera hipótesis, esto es, los hombres presentan mayores niveles de sexismo hostil y benévolo que las mujeres. Estos resultados coinciden con los obtenidos en otras investigaciones (Arbach et al., 2019; De Lemus et al., 2008; Fernández-García et al., 2020; Glick et al., 2000; León \& Aizpurúa, 2020; Zubieta et al., 2011) y sugieren que, de acuerdo con en la Teoría del Sexismo Ambivalente de Glick y Fiske (1996), Ios hombres pretenden mantener su posición de poder frente a las mujeres, lo que explicaría que, por una parte, presenten actitudes benévolas ante las mujeres que cumplen con roles convencionales, reforzando estos comportamientos en ellas; y, por otra parte, muestren actitudes de rechazo ante aquellas que no cumplan con estos estereotipos, debido a que estas últimas podrían ser percibidas como una amenaza frente a la posición dominante del 
hombre. De esta forma ambos tipos de sexismo podrían funcionar como un medio de control de las mujeres (Lee et al., 2010). Así mismo, tanto hombres como mujeres puntuaron más alto en sexismo benévolo que en sexismo hostil, lo que podría deberse a que son expresiones sexistas más sutiles y, quizá por ello están menos asociadas con el sexismo. Estos hallazgos reflejan la persistencia del sexismo, los prejuicios y estereotipos de género en la actualidad, manteniéndose las desigualdades sociales, a pesar de las diversas acciones políticas que se han realizado en España con la finalidad de conseguir la igualdad de género. Por otro lado, en relación con la autoestima sexual no se han hallado diferencias significativas por razón de género al igual que en otros estudios (Amos \& McCabe, 2015; Snell \& Papini, 1989).

Por lo que respecta al segundo objetivo de la investigación, los resultados encontrados no permiten concluir que exista relación entre las creencias sexistas y la autoestima sexual en ambos géneros, ya que únicamente se encontraron correlaciones estadísticamente significativas entre los participantes de género masculino. Así pues, en el presente estudio parece que los hombres con mayor autoestima sexual presentan mayor sexismo benévolo. Estos resultados podrían explicarse por la relación que han obtenido algunas investigaciones entre el sexismo benévolo y las relaciones románticas. Así pues, los hombres con alta confianza en su capacidad de complacer sexualmente a la pareja podrían expresar afecto hacia las mujeres por el deseo de intimar con ellas (Lee et al., 2010), ya que, según los hallazgos de Bohner et al. (2010), las mujeres podrían sentir mayor simpatía y atracción sexual por hombres con actitudes sexistas de tipo benévolo, en comparación con aquellos que presentan actitudes de tipo sexista hostil y no sexista, lo que podría reforzar este tipo de actitudes en ellos. Estos resultados son contrarios a los hallados en otras investigaciones (Fowers \& Fowers, 2010; Sibley \& Wilson, 2004) lo que podría deberse a las características sociodemográficas y culturales de los participantes de las investigaciones citadas, pues en ambos casos la muestra empleada estuvo formada por estudiantes universitarios estadounidenses y neozelandeses.

Respecto al tercer objetivo, los resultados sugieren que la confianza propia sobre la capacidad de complacer a la pareja sexual podría ser un buen predictor del sexismo benévolo únicamente en hombres. Más concretamente, la autoestima sexual explicó el 13,1\% de la variabilidad del sexismo benévolo en este género. Esto es especialmente relevante pues estas actitudes contribuyen al mantenimiento de la posición dominante del género masculino (Lee et al., 2010) y, además, son generalmente más aceptadas por la población (Novo et al., 2016), ya que se subestima el impacto emocional del mismo (Bosson et al., 2010).

\section{CONCLUSIONES}

En definitiva, nuestro trabajo parece señalar que, si bien parece que los niveles de sexismo hostil han disminuido lo que podría ser consecuencia del rechazo social que generan, las actitudes sexistas continúan presentes adquiriendo un tono más afectivo. Por otra parte, este estudio sugiere que podría existir una relación entre la autoestima sexual y el sexismo, en la medida en la que este tipo de actitudes positivas podrían aumentar la posibilidad de establecer relaciones de intimidad con el otro género. Así pues, es necesario mencionar que este trabajo presenta ciertas limitaciones, pues hay variables sociodemográficas que no se han tenido en cuenta como el hecho de tener pareja. Por último, en futuras investigaciones convendría analizar la relación entre el sexismo y otros aspectos de la sexualidad como la satisfacción sexual y el número de parejas sexuales para aportar una mayor claridad sobre la relación entre el sexismo y la sexualidad. 


\section{¿EL SEXISMO Y LA AUTOESTIMA SEXUAL GUARDAN RELACIÓN? ANÁLISIS DE LAS DIFERENCIAS DE GÉNERO}

\section{REFERENCIAS BIBLIOGRÁFICAS}

Amos, N., \& McCabe, M. P. (2015). Conceptualizing and measuring perceptions of sexual attractiveness: Are there differences across gender and sexual orientation? Personality and Individual Differences, 76, 111-122. https://doi.org/10.1016/j.paid.2014.11.057

Arbach, K., Vaiman, M., Bobbio, A., Bruera, J., \& Lumello, A. (2019). Inventario de Sexismo Ambivalente: Invarianza factorial entre géneros y relación con la violencia de pareja. Interdisciplinaria: Revista de Psicología y Ciencias Afines, 36(1), 59-76. https://doi.org/10.16888/interd.36.1.5

Bohner, G., Ahlborn, K., \& Steiner, R. (2010). How sexy are sexist men? Women's perception of male response profiles in the ambivalent sexism inventory. Sex Roles, 62(7-8), 568-582. https://doi.org/10.1007/s11199-009-9665-x

Bosson, J. K., Pinel, E. C., \& Vandello, J. A. (2010). The emotional impact of ambivalent sexism: Forecasts versus real experiences. Sex Roles, 62(7-8), 520-531. https://doi.org/10.1007/s11199-009-9664-y

De Lemus, S., Castillo, M., Moya, M., Padilla, J. L., \& Ryan, E. (2008). Elaboración y validación del inventario de sexismo. Ambivalente para adolescentes. International Journal of Clinical and Health Psychology, 8(2), 537-562.

Eagly, A. H. (1987). Sex Differences in Social Behavior: A Social-role interpretation . Lawrence Erlbaum Associates. https://books.google.es/books?hl=es\&lr=\&id=sf8oxcuiSlsC\&oi=fnd\&pg=PR3\&ots=5yzhwl2bs Y $\&$ sig=bza5SNywszDIQcr4Zg3NG7J8CDE\&redir_esc=y\#v=onepage \&q\&f=false

Fernández-García, 0., Gil-Llario, M. D., \& Ballester-arnal, R. (2020). Prevalencia y caracterización del sexismo en el contexto español. $7^{0}$ Congresso Internacional em Saúde. Braga: 7-9 de octubre de 2020.

Fowers, A. F., \& Fowers, B. J. (2010). Social dominance and sexual self-schema as moderators of sexist reactions to female subtypes. Sex Roles, 62(7-8), 468-480. https://doi.org/10.1007/s11199-009-9607-7

Glick, P., \& Fiske, S. T. (1996). The ambivalent sexism inventory: Differentiating Hostile and Benevolent Sexism. Journal of Personality and Social Psychology, 70(3), 491-512. https://doi.org/DOI: 10.1037/0022-3514.70.3.491N

Glick, P., \& Fiske, S. T. (2001). An ambivalent alliance: Hostile and benevolent sexism as complementary justifications for gender inequality. American Psychologist, 56(2), 109-118. https://doi.org/10.1037/0003-066X.56.2.109

Glick, P., Fiske, S. T., Mladinic, A., Saiz, J. L., Abrams, D., Masser, B., Adetoun, B., Osagie, J. E., Akande, A., Alao, A., Brunner, A., Willemsen, T. M., Chipeta, K., Dardenne, B., Dijksterhuis, A., Wigboldus, D., Eckes, T., Six-Materna, I., Expósito, F., ... López, W. L. (2000). Beyond prejudice as simple antipathy: Hostile and benevolent sexism across cultures. Journal of Personality and Social Psychology, 79(5), 763-775. https://doi.org/10.1037/0022-3514.79.5.763

González-Rivera, I., \& Díaz-Loving, R. (2018). Predictores del Sexismo Ambivalente hacia los Hombres. Acta de Investigación Psicológica, 8(3), 43-51. https://doi.org/10.22201/fpsi.20074719e.2018.3.05

Lee, T. L., Fiske, S. T., \& Glick, P. (2010). Next gen ambivalent sexism: Converging correlates, causality in context, and converse causality, an introduction to the special issue. Sex Roles, 62(7), 395404. https://doi.org/10.1007/s11199-010-9747-9

León, C. M., \& Aizpurúa, E. (2020). Do sexist attitudes persist in college students? An analysis of its prevalence, predictors, and gender differences. Educacion XX1, 23(1), 275-296. https://doi.org/10.5944/educxx1.23629 
Macrae, C. N., Milne, A. B., \& Bodenhausen, G. V. (1994). Stereotypes as energysaving devices: A peek inside the cognitive toolbox. Journal of Personality and Social Psychology, 66(1), 37-47.

Martínez, C. A. (2018). Estereotipos de género y evaluación de candidatos en Estados Unidos: evidencia de una encuesta-experimento Gender stereotypes and candidate evaluation in the United States: evidence from a survey-experiment Estereotipos de genero e evaluacao de candidatos. Psicologia, Conocimiento y Sociedad, 8(1), 36-71. http://dx.doi.org/10.26864/pcs.v8.n1.3

Novo, M., Herbón, J., \& Amado, B. G. (2016). Género y victimización: Efectos en la evaluación de la violencia psicológica sutil y manifiesta, apego adulto y tácticas de resolución de conflictos. Revista Iberoamericana de Psicologia y Salud, 7(2), 89-97. https://doi.org/10.1016/j.rips.2016.05.002

Pai, H. C., Lee, S., \& Yen, W. J. (2012). The effect of sexual self-concept on sexual health behavioural intentions: A test of moderating mechanisms in early adolescent girls. Journal of Advanced Nursing, 68(1), 47-55. https://doi.org/10.1111/j.1365-2648.2011.05710.x

Ramiro-Sánchez, T., Ramiro, M. T., Bermúdez, M. P., \& Buela-Casal, G. (2018). Sexism and sexual risk behavior in adolescents: Gender differences. International Journal of Clinical and Health Psychology, 18(3), 245-253. https://doi.org/10.1016/j.ijchp.2018.04.002

Sibley, C. G., \& Wilson, M. S. (2004). Differentiating Hostile and Benevolent Sexist Attitudes Toward Positive and Negative Sexual Female Subtypes 1. Sex Roles, 51. https://doi.org/10.1007/s11199-004-0718-x

Snell, W. E., Fisher, T. D., \& Walters, A. S. (1993). The multidimensional sexuality questionnaire: An objective self-report measure of psychological tendencies associated with human sexuality. Annals of Sex Research, 6(1), 27-55. https://doi.org/10.1007/BF00849744

Snell, W. E., \& Papini, D. R. (1989). The sexuality scale: An instrument to measure sexual-esteem, sexual-depression, and sexual-preoccupation. The Journal of Sex Research, 26(2), 256-263. https://doi.org/10.1080/00224498909551510

Soto-Quevedo, 0. (2012). The role of ambivalent sexism and transgression of gender stereotype on attribution of blame to female victims of intimate partner violence. ACTA COLOMBIANA DE PSICOLOGÍA, 15(2), 135-147. http://www.scielo.org.co/pdf/acp/v15n2/v15n2a13.pdf

Torres-Obregon, R., Onofre-Rodríguez, D. J., Sierra, J. C., Granados, R., Garza-Elizondo, M. E., \& Benavides-Torres, R. A. (2019). Interpersonal factors as predictors of sexual self-esteem in women in Northeast Mexico. Revista Internacional de Andrologia, 17(2), 55-59. https://doi.org/10.1016/j.androl.2018.03.003

Zubieta, E., Beramendi, M., Sosa, F., \& Torres, J. A. (2011). Sexismo ambivalente, estereotipos y valores en el ámbito militar. Revista de Psicología (PUCP), 29(1), 101-130. http://www.scielo.org.pe/scielo.php?script=sci_arttext\&pid=S025492472011000100004\&lng=es\&tlng=es. 
